\title{
Tratamiento endovascular de la disección aórtica tipo B mediante endoprótesis
}

\author{
Renato Mertens $\mathrm{M}^{1}$, Ivette Arriagada J, Francisco Valdés $\mathrm{E}^{1}$, \\ Albrecht Krämer $\operatorname{Sch}^{1}$, Leopoldo Mariné $M^{1}$, \\ Michel Bergoeing $\mathbf{R}^{1}$, Sandra Braun J ${ }^{2}$, Iván G odoy J2, \\ Samuel Córdova $A^{2}$, Alvaro H uete $\mathbf{G}^{3}$, Jeannette Vergara $G^{1 a}$, \\ Claudia Carvajal Na.
}

\section{Endovascular treatment of type $B$ aortic dissection}

Background: Dissections that involve the ascending aorta are classified as type A, regardless of the site of the primary intimal tear, and all other dissections as type B. Type B dissections can have fatal ischemic and hemorrhagic complications. In the chronic state, dilatation and rupture can be mortal. Endovascular surgery is a therapeutic alternative, considering the high rate of complications of conventional surgery. Aim: To report the results of endovascular treatment of type B aortic dissection. Material and methods: Report of 36 treated patients (30 males) aged 43 to 87 years, with a type B aortic dissection. Seventy eight percent were hypertensive and $39 \%$ smoked. The diagnosis was confirmed by CAT scan. Acute patients were treated for complications and chronic patients, for dilatation. In the operating room, an endoprothesis was placed through the femoral artery, to cover the tear. The tear was located and the lumens were differentiated using angiography and transesophageal echocardiography. Results: All procedures were successful. In 16 acute dissections the indications were malperfusion syndrome or unmanageable hypertension in seven patients and imminent rupture or persistent pain in nine. Twenty chronic patients were operated due to dilatation (mean $6 \mathrm{~cm}$ ). One patient died due to cardiac failure. One patient had a transient paraparesia and two had pulmonary embolism. No patient died in a follow up period ranging from 2.5 to 74 months. Four patients required a new aortic endovascular procedure due to progressive dilatation or endoleak. Conclusion: Endovascular treatment of type B aortic dissection has good immediate and long term results (Rev Méd Chile 2008; 136: 1431-8).

(Key w ords: Aortic aneurism; Aortic dissection; Stents)

Recibido el 24 de abril, 2008. Aceptado el 29 de julio, 2008.

Departamento de Cirugía Vascular y Endovascular, División de Cirugía ${ }^{1}$, Departamentos de Enfermedades Cardiovasculares ${ }^{2}$ y de Radiología ${ }^{3}$, Pontificia Universidad Católica de Chile. aEnfermera Universitaria

Correspondencia a: Dr. Renato Mertens M. Apoquindo

3990, oficina 601, Santiago. Fono: (56 2) 207 0721. Fax: (56

2) 207 0718. E mail: rmertens@manquehue.net 
La disección aórtica tipo $\mathrm{B}$ consiste en el desgarro de la íntima de la aorta torácica descendente, habitualmente inmediatamente distal al nacimiento de la arteria subclavia izquierda, con la formación de un canal paralelo al lumen natural denominado lumen falso. Este último se propaga hacia distal y puede producir compresión del lumen verdadero, causando estenosis u obstrucción de ramas que nacen de la aorta al avanzar hacia éstas y generando el debilitamiento de la pared, facilitando la ruptura aguda o la formación de un aneurisma en la etapa crónica.

En los últimos años el desarrollo de técnicas endovasculares mínimamente invasivas ha permitido el tratamiento exitoso de múltiples patologías graves de la aorta torácica y abdominal que de otra manera requerirían de extensos procedimientos quirúrgicos ${ }^{1-5}$.

En 1999, simultáneamente los doctores Dake, de Estados Unidos de Norteamérica, y Nienaber, de Alemania, reportaron la posibilidad de aplicar el uso de una endoprótesis para tratar la disección aórtica aguda complicada ${ }^{6,7}$. Sus resultados fueron alentadores en una patología donde la cirugía tradicional ofrece malos resultados ${ }^{8}$. Por otro lado, al menos en los pacientes sin complicaciones el tratamiento médico presenta buenos resulta$\operatorname{dos}^{9,10}$.

Comunicamos nuestra experiencia en el tratamiento endovascular de esta grave condición.

\section{MATERIAL Y MÉTODO}

En el año 2001 iniciamos un protocolo prospectivo para el tratamiento endovascular de patología de la aorta torácica descendente y tóraco-abdominal, incorporando a pacientes portadores de disección aórtica tipo B en su fase aguda y crónica, definiendo arbitrariamente la diferencia entre ambas, los 30 días de evolución.

El diagnóstico estuvo basado en un cuadro clínico compatible y la obtención de imágenes mediante ecocardiografía transesofágica o tomografía axial computada.

Las indicaciones para este tratamiento en la etapa aguda se dividieron en dos grupos. El primero constituido por pacientes con amenaza de ruptura aórtica, definida como crecimiento rápido del diámetro aórtico, dolor torácico persis- tente, derrame pleural en aumento o hematoma periaórtico. El segundo grupo es conformado por pacientes con síndrome de malaperfusión, definido como el colapso del lumen verdadero y la disminución de la perfusión renal, visceral o de las extremidades inferiores. No se trataron en la etapa aguda pacientes con buena respuesta al tratamiento médico. En la etapa crónica se indicó el procedimiento en pacientes portadores de dilatación aneurismática mayor a $5 \mathrm{~cm}$.

Previo al procedimiento, todos los pacientes fueron evaluados mediante tomografía axial computada helicoidal multicorte con contraste en fase arterial, obteniendo reconstrucciones axiales cada 3,75 mm o menos, con la posibilidad de obtener reconstrucciones coronales o sagitales.

Los criterios para ser incluidos en el protocolo endovascular fueron:

1. Factibilidad de acceso a través de las arterias femorales o ilíacas para introducir un dispositivo de alto diámetro, pudiendo llegar a los $26 \mathrm{~F}$.

2. Presencia de un segmento no comprometido de aorta para la fijación proximal del dispositivo de al menos $15 \mathrm{~mm}$ inmediatamente distal al origen de la arteria carótida común izquierda en el arco aórtico, considerando la posibilidad de ocluir el origen de la arteria subclavia izquierda en forma programada en caso de requerirlo.

3. La posibilidad de iniciar un protocolo de seguimiento a largo plazo.

4. La disponibilidad inmediata del dispositivo en los casos agudos y la accesibilidad diferida en los casos crónicos.

La evaluación preoperatoria incluyó los parámetros básicos necesarios para un procedimiento de esta envergadura.

Todos los procedimientos fueron realizados en pabellón quirúrgico o sala de angiografía, bajo anestesia general y monitorización invasiva.

Para la generación de las imágenes intraoperatorias se utilizó en la sala de operaciones un angiógrafo digital portátil Siremobil (Siemens ${ }^{\circledR}$, Alemania) u OEC serie 9.800 (General Electric ${ }^{\circledR}$, EE.UU.).

La prótesis fue insertada previa denudación y arteriotomía de la arteria femoral común seguida de su reparación quirúrgica. Esto último debido a que el alto diámetro del sistema introductor del dispositivo impide su uso percutáneo. 
El procedimiento fue realizado bajo heparinización sistémica, manteniendo el tiempo de coagulación activado (ACT) sobre 300 s. Se utilizó radioscopia, angiografía por sustracción digital y esencialmente apoyo con ecocardiografía transesofágica para orientar y desplegar la endoprótesis en el lugar apropiado, en particular en la definición de los lúmenes verdadero y falso de la lesión y el o los lugares de comunicación entre ambos.

La mortalidad operatoria fue definida como intrahospitalaria o antes de 30 días.

Endofuga o endoleak fue definida como persistencia de flujo hacia el falso lumen, detectada mediante tomografía axial computada. Utilizamos la nomenclatura habitual reconocida para este tipo de complicaciones: Tipo I son filtraciones a través del sitio de anclaje proximal de la endoprótesis en la aorta, las tipo II son dependientes de ramas que se originan del segmento de aorta tratado, tipo III se producen por falla estructural de la endoprótesis.

Exito técnico fue definido como el despliegue de la endoprótesis en el lumen verdadero, cierre de la comunicación más proximal entre el lumen verdadero y falso y la inducción de trombosis del

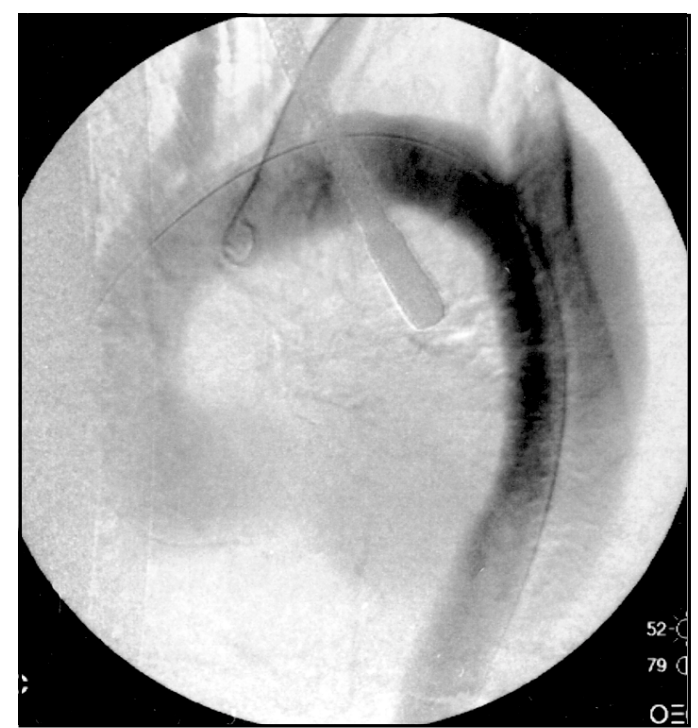

Figura 1. Angiografía con inyección de contraste en el arco aórtico previa al despliegue de la endoprótesis. Se observa llenado inmediato del falso lumen. falso lumen a nivel torácico en relación con la endoprótesis (Figuras 1 y 2).

Previo al alta se realizó una tomografía axial computada helicoidal con contraste en fase arterial para la evaluación de la localización de la endoprótesis y la presencia de endofugas. Si la tomografía resultaba satisfactoria se programaba un control a los 6 y 12 meses, luego anualmente.

\section{RESULTADOS}

Entre octubre de 2001 y agosto de 2007, 36 pacientes consecutivos, portadores de disección aórtica tipo $B$, fueron tratados con endoprótesis torácica, previo consentimiento informado. El promedio de edad fue 60,9 años (rango 43-87). El $83 \%$ ( $n=30)$ eran hombres. Las comorbilidades se detallan en la Tabla 1, las más frecuentes son la hipertensión arterial (77,8\%), el tabaquismo $(38,9 \%)$ y la dislipidemia (25\%).

En todos los pacientes, el diagnóstico de disección aórtica se hizo por tomografía computada.

En 16 pacientes, la indicación de endoprótesis fue la presencia de complicación de una disección

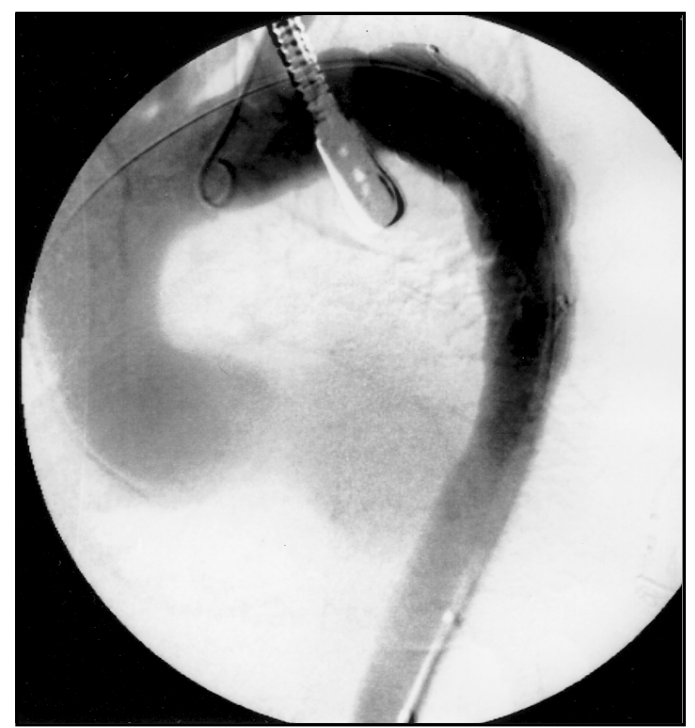

Figura 2. Angiografía con inyección de contraste en el arco aórtico posterior al despliegue de la endoprótesis. Se visualiza llenado del lumen verdadero con exclusión del falso lumen. 
Tabla 1. Patologías asociadas en los 36 pacientes tratados por disección de aorta torácica tipo B entre octubre de 2001 y agosto de 2007

\begin{tabular}{|lrr|}
\hline Comorbilidad & $\%$ & $\mathbf{n}$ \\
\hline Hipertensión arterial & 77,8 & 28 \\
Tabaquismo & 38,9 & 14 \\
Dislipidemia & 25,0 & 9 \\
Obesidad & 11,1 & 4 \\
Insuficiencia renal crónica & 8,3 & 3 \\
Enfermedad de Marfan & 8,3 & 3 \\
Cardiopatía coronaria & 5,6 & 2 \\
\hline
\end{tabular}

aórtica aguda complicada. En 9 de estos pacientes por ruptura inminente debido a dilatación aguda 0 dolor persistente y en 7 pacientes, por hipertensión arterial inmanejable o síndrome de malperfusión. Las indicaciones se detallan en la Tabla 2.

La indicación de endoprótesis fue la dilatación aneurismática mayor a $5 \mathrm{~cm}$ por disección aórtica crónica en 20 de los pacientes. El diámetro promedio del aneurisma en estos pacientes fue de $6 \mathrm{~cm}$.

Las endoprótesis utilizadas se describen en la Tabla 3, utilizándose sólo dispositivos comencialmente disponibles.

En el intraoperatorio se produjo un desgarro de la arteria femoral en un paciente, que se solucionó localmente mediante un puente arterial. En el periodo de postoperatorio precoz, se registraron 2 casos de embolia pulmonar demostrados por tomografía axial computada (5,5\%). No hubo falla renal ni respiratoria asociada.

En todos los pacientes se obtuvo mejoría sintomática. No hubo conversión a cirugía abierta. Éxito técnico en $100 \%$ de los pacientes.

La hospitalización postoperatoria se extendió, en promedio, por 6,8 días (rango 1 a 64, DE 10,9). Fue necesario transfundir, entre 2 y 4 unidades de glóbulos rojos a sólo 5 pacientes (13,9\%).

No se registraron casos de accidente cerebrovascular. Hubo un paciente $(2,8 \%)$ que presentó paraplegia en el postoperatorio inmediato. Tuvo buena respuesta al tratamiento inmediato con reperfusión vertebral mediante stent de la arteria subclavia izquierda, la que había sido cubierta intencionalmente con la endoprótesis, drenaje espinal para disminuir la presión del líquido cefalorraquídeo, corticoides y kinesioterapia, re-

\section{Tabla 2. Indicación de endoprótesis en 36 pacientes tratados por disección aorta torácica tipo B entre octubre de 2001 y agosto de 2007}

\begin{tabular}{|lcc|}
\hline Indicación & $\mathbf{n}$ & $\%$ \\
\hline Disección aórtica crónica con dilatación aneurismática $>5 \mathrm{~cm}$ & 20 & 55,6 \\
& & \\
Complicación de disección aórtica aguda & 16 & 19,4 \\
Pseudocoartación & 7 & 11,1 \\
Úlcera penetrante con disección localizada y dolor & 4 & 11,1 \\
Dilatación aguda & 4 & 2,8 \\
Hematoma periaórtico - derrame pleural izquierdo & 1 & \\
\hline
\end{tabular}




\section{Tabla 3. Prótesis utilizadas en 36 pacientes tratados por disección de aorta torácica tipo B entre octubre de 2001 y agosto de 2007}

\begin{tabular}{|lrr|}
\hline Endoprótesis utilizada & $\%$ & $\mathbf{n}$ \\
\hline Talent/Valiant $\left(\right.$ Medtronic $^{\circledR}$, EE.UU.) & 41,7 & 15 \\
TX2 (COOK ${ }^{\circledR}$ EE.UU.) & 38,9 & 14 \\
TAG (Goretex ${ }^{\circledR}$, EE.UU.) & 19,4 & 7 \\
\hline
\end{tabular}

cuperándose hasta la deambulación sin ayuda, control esfinteriano completo y reintegración laboral, pero con una paresia leve persistente.

La mortalidad perioperatoria fue de $2,8 \%$, que correspondió a una paciente que presentó un infarto al miocardio antes de $24 \mathrm{~h}$ demostrado por necropsia.

Se obtuvo seguimiento clínico en $100 \%$ de los pacientes por un periodo promedio de 30 meses (rango 2,5-74,3).

Seguimiento imagenológico con tomografía computada o ultrasonografía transesofágica se obtuvo en $80 \%$ de los pacientes al momento del cierre del estudio, por un periodo promedio de 21 meses (rango 0,1-74,3). En 100\% de estos pacientes se ha logrado la trombosis del falso lumen a nivel de la endoprótesis.

Se han reintervenido 5 pacientes. Cuatro de ellos por desarrollo de una endofuga tipo I, en 3 de ellos el tratamiento consistió en la extensión de la endoprótesis insertando un nuevo segmento y en uno de ellos en la embolización de la endofuga. Un paciente requirió de la instalación de un stent de arteria renal por el desarrollo de isquemia renal secundaria a la progresión de la disección.

Cuatro pacientes presentan endofuga tipo II, sin desarrollo de dilatación aórtica. No han sido intervenidos y se mantienen en seguimiento. No hemos observado casos de migración ni de fractura de la endoprótesis.

No ha habido mortalidad tardía.

\section{Discusión}

La disección aórtica tipo B es una enfermedad grave que puede seguir múltiples cursos clínicos ${ }^{8}$. En la mayoría de los casos el paciente debuta sólo con dolor torácico intenso pero transitorio y con manejo médico de la hipertensión, los resultados inmediatos son buenos, siendo necesario el seguimiento con imágenes para detección precoz de la principal complicación en la etapa crónica: la dilatación y formación de un aneurisma ${ }^{9,10}$.

Sin embargo, 22\% de los pacientes requieren de intervención aguda por complicaciones ${ }^{8}$, las que pueden dividirse en dos grupos, el primero es la amenaza de ruptura aórtica o ruptura franca, que se manifiesta por dolor persistente, aparición de derrame pleural denso o compromiso hemodinámico por hemorragia (Figura 3). El segundo grupo consiste en complicaciones de tipo isquémico, que pueden ir desde la isquemia localizada de un órgano o extremidad por compresión del lumen de la arteria que lo irriga por avance de la disección, hipertensión intratable por un fenómeno de pseudocoartación dinámica de la aorta, producida por hipertensión del lumen falso y colapso del lumen verdadero intermitentemente durante el ciclo cardíaco o la pseudocoartación permanente, su forma más grave, con isquemia global visceral, renal y de extremidades inferiores (Figura 4).

La sobrevida de los pacientes portadores de una disección tipo B es limitada, 12\% fallece globalmente en el hospital y $28 \%$ fallece antes de un año ${ }^{8}$.

Es el grupo que presenta complicaciones agudas donde el procedimiento endovascular puede resolver en forma expedita y poco invasi$\mathrm{va}^{6,7}$ un problema que la cirugía abierta requeriría de extensos y cruentos procedimientos con una alta morbimortalidad asociada ${ }^{8,11}$.

En la etapa crónica la aorta puede dilatarse y formar un aneurisma que puede llevar a ruptura y muerte, es así como alrededor de un tercio de los pacientes portadores de una disección tipo B crónica desarrollarán un aneurisma. Se han buscado elementos predictivos que puedan sugerir 


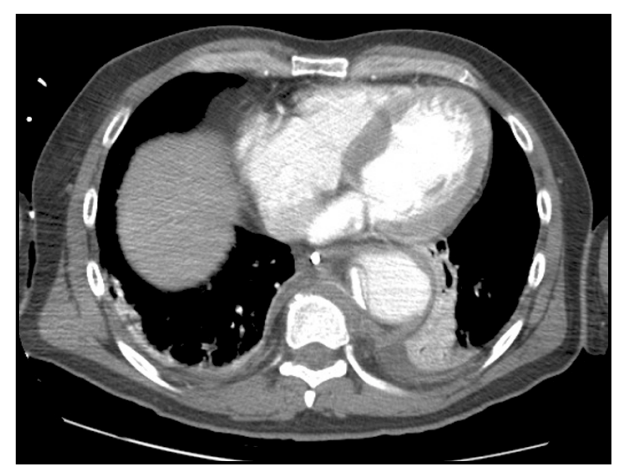

Figura 3. Reconstrucción axial de tomografía axial computada con contraste en fase arterial. Muestra dilatación aguda de la aorta asociada a derrame pleural denso periaórtico en un paciente con dolor torácico persistente.

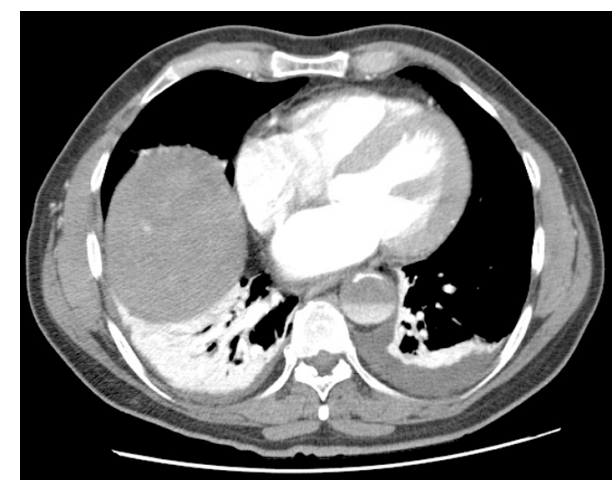

Figura 4a. Reconstrucción axial de tomografía axial computada con contraste en fase arterial. Muestra coartación aguda del lumen verdadero en paciente con isquemia aguda visceral y de extremidades inferiores.

durante la etapa aguda cuál paciente desarrollará un aneurisma posteriormente. La dilatación aguda de la aorta a un diámetro mayor de $4 \mathrm{~cm}$, especialmente si es asociado a persistencia de flujo en el falso lumen es un elemento predictivo importante $^{12}$. La presencia de hipertensión no controlada, síndrome de Marfán y enfermedad pulmonar obstructiva crónica, son también factores independientes que predicen eventual dilatación ${ }^{13}$. Recientemente se ha descrito al diámetro del falso lumen a nivel de la aorta torácica descendente proximal mayor de $22 \mathrm{~mm}$ como un elemento muy sensible y específico para predecir la dilatación ${ }^{14}$. Está pendiente evaluar si acaso el tratamiento endovascular precoz de estos pacientes seleccionados determinará una reducción de la

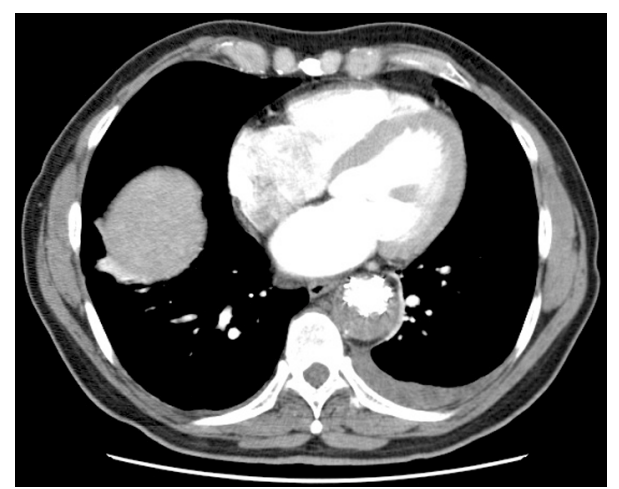

Figura 4b. El mismo paciente luego del despliegue de endoprótesis. Se demuestra ampliación del lumen verdadero y ausencia de flujo en lumen falso.

dilatación progresiva, pero es posible que así sea, por otro lado ese grupo de pacientes es normalmente el que tiene una peor respuesta al tratamiento médico en la etapa aguda, presentándose con dolor persistente o isquemia que finalmente lleva a indicar un procedimiento endovascular precoz.

Consideramos muy satisfactorios los resultados obtenidos en el tratamiento de esta grave patología. En la fase aguda todos los pacientes resolvieron el síntoma que motivó el procedimiento y no hubo mortalidad operatoria. En la etapa crónica, fallece sólo un paciente de una causa no relacionada con la técnica.

El seguimiento con imágenes periódicas es crítico para poder diagnosticar fallas asintomáti- 
cas, antes de que se genere una complicación clínicamente relevante. Esto ha sido demostrado por otros grupos, incluyendo las fallas mecánicas de la endoprótesis, por fractura de los stents metálicos o ruptura de la tela impermeable ${ }^{15}$. Aunque el objetivo terapéutico se obtuvo en todos los pacientes, cuatro han requerido una reintervención aórtica durante el seguimiento alejado, todos tratados exitosamente por vía endovascular. Ningún caso ha sido convertido a cirugía abierta.

Los resultados comunicados por otros grupos han sido también muy satisfactorios. Mostrando resolución de los síntomas en la etapa aguda y eventual remodelación de la aorta torácica, con tendencia a disminución u obliteración del lumen falso y aún más importante que este hecho morfológico, es la baja incidencia de crecimiento o ruptura en la fase crónica ${ }^{16-20}$.

La pregunta obvia es si el resultado es tan favorable, por qué no tratar con endoprótesis a todos los pacientes portadores de disección tipo B en la etapa aguda? Se ha diseñado un estudio prospectivo y randomizado para contestar esta

\section{REFERENCIAS}

1. Mertens R, Valdés F, Kämer A. Tratamiento Endovascular del Aneurisma de Aorta. Revista Chilena de Cirugía 2004; 56: 3-11.

2. Mertens R, Vaidés F, Kä̈mer A, Mariné L, Irarrázaval M, Morán S ET AL. Tratamiento endovascular del aneurisma de aorta torácica descendente. Rev Méd Chile 2003; 131: 390-6.

3. Valdés F, Mertens R, Krämer A, Bergoeing M, Mariné L, Canessa R et al. Tratamiento Endovascular de Aneurisma Aórtico Abdominal: Resultados en 80 Pacientes Consecutivos. Rev Méd Chile 2006; 134: 1265-74.

4. Mertens R, Valdés F, Krämer A, Bergoeing M, ZalaQUETT R, BAEZA C ET AL. Tratamiento endovascular del trauma de aorta descendente. Rev Méd Chile 2005; 133: 403-8.

5. Mertens R, Vaidés F, Kramer A, Mariné L, Bergoeing M, Sagües R et al. Tratamiento Híbrido del Aneurisma Tóraco-Abdominal: Revascularización Extra-Anatómica e Inserción de Endoprótesis. Rev Méd Chile 2007; 135: 153-9.

6. Dake M, Kato N, Mitchell R, Semba C, Razabi M, Shimono T et al. Endovascular stent-graft placement for the treatment of acute aortic dissection. N Engl J Med 1999; 340: 1546-52.

7. Nienaber C, Fattori R, Lund G, Dieckmann C, Wolf W, von Kodolitsch Y ET AL. Nonsurgical reconstruction of pregunta $^{21}$, aunque sus resultados no han sido reportados en la literatura, han sido presentados por nuestro grupo en conferencias, mostrando que la mortalidad es mayor a 12 meses de seguimiento en los pacientes intervenidos que en los tratados médicamente. Por este motivo y mientras no se cuente con información confiable, pensamos que el limitar este procedimiento sólo a los pacientes con complicaciones es lo indicado, esto ha sido confirmado por un reciente consenso de expertos ${ }^{22}$. Así mismo, en dicho trabajo se ha hecho un intento en aclarar algunas definiciones e indicaciones, sin embargo su metodología lamentablemente dista de ser precisa al no contarse con series prospectivas y aleatorias que permitan llegar a conclusiones claras.

Nuestros resultados inmediatos y a mediano plazo con el uso de este procedimiento, asociado a los buenos resultados reportados en la literatura, nos ha permitido considerar a esta técnica como una opción de primera línea en el manejo de pacientes con complicaciones de esta grave patología.

thoracic aortic dissection by stent-graft placement. N Engl J Med 1999; 340: 1539-45.

8. Tsai T, Fattori R, Trimarchi S, Isselbacher E, Myrmel T, Evangelista A ET AL, ON BEHALF OF THE INTERNATIONAL Registry of Acute Aortic Dissection (IRAD). LongTerm Survival in Patients Presenting With Type B Acute Aortic Dissection: Insights From the International Registry of Acute Aortic Dissection. Circulation 2006; 114: 2226-31.

9. WienNerkvist A, Lockowandt U, Rasmussen E, Radegran K. A prospective study of medically treated acute type B aortic dissection. Eur J Vasc Endovasc Surg 2006; 32: 349-55.

10. Estrera A, Miuer C, Safi H, Goodrick J, Keyhani A, Porat E ET AL. Outcomes of medical management of acute type B aortic dissection. Circulation 2006; 114 (1 Suppl): I384-9.

11. Karmy-Jones R, Simeone A, Meissner M, Granvall B, NichoLs S. Descending thoracic aortic dissections. Surg Clin North Am 2007; 87: 1047-86.

12. Marui A, Mochizuki T, Mitsui N, Koyama T, Kimura F, HoriBE M. Toward the best treatment for uncomplicated patients with type B acute aortic dissection: A consideration for sound surgical indication. Circulation 1999; 100(19 Suppl): II275-80.

13. Juvonen T, Ergin M, Galia J, Lansman S, McCulough J, NGUYEN K ET AL. Risk factors for rupture of chronic type B dissections. J Thorac Cardiovasc Surg 1999; 117: 776-86. 
14. Song J, Kim S, Kim J, Kim M, Kang D, Seo J et al. LongTerm Predictors of Descending Aorta Aneurysmal Change in Patients with Aortic Dissection. J Am Coll Cardiol 2007; 50: 799-804.

15. Jacobs T, Won J, Gravereaux E, Faries P, Morrisey N, TEOdorescu V ET AL. Mechanical failure of prosthetic human implants: A 10-year experience with aortic stent graft devices. J Vasc Surg 2003; 37: 16-26.

16. Eggebrecht H, Nienaber C, Neuhäuser M, Baumgart D, Kische S, SCHMERMUND A ET AL. Endovascular stent-graft placement in aortic dissection: a meta-analysis. Eur Heart J 2006; 27: 489-98.

17. Schoder M, Czerny M, Cejna M, Rand T, Stadier A, SODECK G ET AL. Endovascular repair of acute type B aortic dissection: long-term follow-up of true and false lumen diameter changes. Ann Thorac Surg 2007; 83: 1059-66.

18. Resch T, Dele M, Falkenberg M, Ivancev K, Konrad P, LARZON T ET AL. Remodeling of the thoracic aorta after stent grafting of type B dissection: a Swedish multicenter study. J Cardiovasc Surg 2006; 47: 503-8.

19. Xu S, Huang F, Yang J, Li Z, Wang X, AhZng Z et al. Endovascular repair of acute type $B$ aortic dissection: early and mid-term results. J Vasc Surg 2006; 43: 1090-5.

20. Kusagana H, Shimono T, Ishida M, Suzuki T, Yasuda F, YuASA U ET AL. Changes in False Lumen After Transluminal Stent-Graft Placement in Aortic Dissections. Six Years' Experience. Circulation 2005; 111: 2951-57.

21. Nienaber C, Zannetti S, Barbieri B, Kische S, Schareck W, REHDERS T, INSTEAD STUDY COLABORATORS. Investigation of stent grafts in patients with type B Aortic Dissection: design of the instead trial-a prospective, multicenter, European randomized trial. Am Heart J 2005; 149: 592-9.

22. Svensson L, Kouchoukos N, Miler G. Expert Consensus Document on the Treatment of Descending Thoracic Aortic Disease Using Endovascular StentGrafts. Ann Thorac Surg 2008; 85: S1-41. 